\title{
Estudo retrospectivo das neoplasias da cavidade oral diagnosticadas entre 1974 e 1995 pelo Serviço de Patologia do Hospital Veterinário da Universidade Federal do Paraná, Curitiba, PR, Brasil
}

\section{Retrospective study of neoplasms of the oral cavity diagnosed be- tween 1974 and 1995 at the Service of Animal Pathology, Veterinary Hospital, Federal University of Paraná, Curitiba, PR, Brazil}

\author{
Pedro Ribas Werner ${ }^{1}$, Márcio Chiquito ${ }^{2}$, José Ricardo Pachaly ${ }^{3}$
}

\section{Resumo}

De abril de 1974 a dezembro de 1995, dentre 960 casos de neoplasias diagnosticados em animais no Serviço de Patologia do Hospital Veterinário da Universidade Federal do Paraná, 48 (5\%) eram de neoplasias localizadas na cavidade oral de 31 cães, 13 bovinos, 1 gato, 1 galinha, 1 leão e 1 papagaio. Destes, 35 casos (72,9\%) eram de neoplasias malignas, sendo 5 carcinomas espinocelulares, 5 hemangiossarcomas, 3 fibrossarcomas, 2 melanocarcinomas, 1 linfossarcoma, 1 mastocitoma, 1 carcinóide, 1 adenocarcinoma de glândula mucosa e 1 carcinoma indiferenciado. As neoplasias benignas totalizaram 13 casos $(27,1 \%)$, sendo que 5 deles representavam neoplasias consideradas como localmente agressivas ( 3 casos de epúlide acantomatosa e 3 de ameloblastoma). As outras neoplasias benignas diagnosticadas foram 5 casos de epúlide fibromatosa e 3 casos de papiloma.

Palavras chave: neoplasia; tumores da cavidade oral; patologia veterinária

\section{Introdução}

A importância clínico-patológica das neoplasias da cavidade oral reside em três fatores. Primeiramente, a cavidade oral é o sítio de $5,4 \%$ das neoplasias malignas dos cães e de $6,7 \%$ das dos gatos (Maretta et al., 1990). Em termos de neoplasias sólidas, só a pele e as glândulas mamárias apresentam incidência maior (White, 1991). Um estudo realizado no Pará, abrangendo 74 cães e 1 gato, constatou que somente a cadeia mamária, os órgãos genitais e a pele apresentavam maior incidência de neoplasias que a cavidade oral (Bittencourt et al., 1994). $\mathrm{Em}$ segundo lugar, de um modo geral, os tumores orais malignos, além de muito mais comuns que os benignos, são muito agressivos (Maretta et al., 1990). Seu prognóstico vai de reservado, para o carcinoma espinocelular, a plenamente desfavorável, para o melanocarcinoma. Mesmo entre os tumores não malignos, com prognóstico favorável, há alguns muito destrutivos, como a epúlide acantomatosa (White, 1991). Finalmente, os tumores orais malignos, em suas fases iniciais, não causam sinais clínicos. Além disso, os proprietários não têm o costume de examinar a boca dos animais e, por isso, o paciente portador de neoplasia oral sofre exame médico apenas quando o tumor já está avançado (Theilen e Madewell, 1987a, b).

Assim, os tumores da cavidade oral têm sido alvo de vários estudos a respeito de seu comportamento biológico, clínico e epidemiológico. Este trabalho estuda, retrospectivamente, a ocorrência de neoplasias orais em animais da região de Curitiba, Paraná, entre abril de 1974 e dezembro de 1995. A prevalência dessas neoplasias é analisada estatisticamente e discutida diante da literatura.

\section{Material e Método}

Empregaram-se os dados dos arquivos do Serviço de Patologia do Hospital Veterinário da Universidade Federal do Paraná (HV-UFPR) referentes aos casos de neoplasias da cavidade oral de animais. Esses arquivos contêm registros de diagnósticos histopatológicos desde abril de 1974. As peças de biópsia ou necropsia referentes às neoplasias em questão foram enviadas àquele Serviço por veterinários do HV-UFPR e da iniciativa privada de Curitiba e região metropolitana.

Os dados recolhidos desses registros foram tabulados e os cortes histológicos de neoplasias orais foram revisados para adaptar os diagnósticos à terminologia mais recente ou aceita. Havendo cinco ou mais casos de

\footnotetext{
1 Patologia Animal, Departamento de Medicina Veterinária, Setor de Ciências Agrárias, Universidade Federal do Paraná (UFPR), Caixa Postal 2959, 80001-970 Curitiba,PR, Brasil

2 Médico Veterinário Autônomo em Curitiba, PR

${ }_{3}^{3}$ Clínica de Animais Selvagens e de Zoológico, Departamento de Medicina Veterinária, Setor de Ciências Agrárias, UFPR
} 
um mesmo tipo histológico de neoplasia, os dados eram colocados numa tabela analisando-se sua ocorrência segundo a idade, o sexo e a raça dos pacientes.

As neoplasias foram classificadas em benignas e malignas. A epúlide acantomatosa e o ameloblastoma foram enquadrados na categoria especial de neoplasias benignas localmente agressivas.

\section{Resultados}

Dentre um total de 960 neoplasias, confirmadas por diagnóstico histológico desde abril de 1974, havia 48 (5\%) neoplasias orais. A distribuição destas entre as espécies é apresentada na Tabela 1.

Tabela 1 - Distribuição, segundo a espécie, dos casos de neoplasia oral diagnosticados no Serviço de Patologia Animal do Hospital Veterinário da Universidade Federal do Paraná entre abril de 1974 e dezembro de 1995

\begin{tabular}{lcc}
\hline Espécie animal & $\begin{array}{c}\text { Número } \\
\text { de casos }\end{array}$ & Percentagem \\
\hline Cão (Canis familiaris) & 31 & 64,5 \\
Bovinos (Bos taurus \& B. indicus) & 13 & 27,1 \\
Gato (Felis catus) & 1 & 2,1 \\
Galinha (Gallus gallus) & 1 & 2,1 \\
Leão (Panthera leo) & 1 & 2,1 \\
Papagaio (Amazona sp.) & 1 & 2,1 \\
\hline Total & 48 & 100 \\
\hline
\end{tabular}

Neoplasias benignas - As neoplasias benignas totalizaram 13 casos, ou $27,1 \%$ do total das neoplasias orais. Havia registros de 5 casos de epúlide fibromatosa e 3 casos de papiloma. As neoplasias benignas localmente agressivas foram representadas por 3 casos de epúlide acantomatosa e 2 de ameloblastoma. A Tabela 2 apresenta a distribuição das neoplasias benignas pelas espécies.

Tabela 2 - Distribuição, segundo a espécie animal, dos casos de neoplasias orais benignas diagnosticadas pelo Serviço de Patologia do Hospital Veterinário da Universidade Federal do Paraná entre abril de 1974 e dezembro de 1995

\begin{tabular}{lccccc}
\hline & \multicolumn{4}{c}{ Espécie animal } & Total \\
\cline { 2 - 5 } Tipo histológico & Cão & Bovino & Leão & Gato & \\
\hline Epúlide fibromatosa & 5 & - & - & - & 5 \\
Papiloma & 1 & 1 & 1 & - & 3 \\
Epúlide acantomatosa & 3 & - & - & - & 3 \\
Ameloblastoma & 1 & - & - & 1 & 2 \\
\hline Total & 10 & 1 & 1 & 1 & 13 \\
\hline
\end{tabular}

Epúlide fibromatosa - Foram diagnosticados 5 casos, todos em cães. A distribuição segundo a raça e o sexo é mostrada na Tabela 3. Os pacientes eram 2 Boxers e 2 cães sem raça definida (SRD), sendo 2 machos e uma fêmea. $O$ registro não mencionava a raça e o sexo do quinto animal. Todos esses tumores eram gengivais. A idade dos animais variou entre 8 e 15 anos. A epúlide fibromatosa coexistia com mastocitoma extra-oral, na fêmea Boxer e com quimiodectoma de base cardíaca, no Boxer macho.

Tabela 3 - Distribuição, segundo raça e sexo, dos casos de epúlide fibromatosa diagnosticados em cães pelo Serviço de Patologia do Hospital Veterinário da Universidade Federal do Paraná, entre abril de 1974 e dezembro de 1995

\begin{tabular}{lcccc}
\hline & \multicolumn{3}{c}{ Raça } & Total \\
\cline { 2 - 4 } Sexo & Boxer & SRD & NE & \\
\hline Machos & 1 & 2 & - & 3 \\
Fêmeas & 1 & - & - & 1 \\
NE & - & - & 1 & 1 \\
\hline Total & 2 & 2 & 1 & 5 \\
\hline
\end{tabular}

SRD: sem raça definida; NE: não especificado

Papiloma - Foram registrados 3 casos, sendo 1 na cavidade oral de um cão Pequinês, macho, com 1 ano de idade, 1 na língua de uma vaca Charolês, de 4 anos, e 1 na língua de um leão (Panthera leo), macho, com 12 anos de idade, e que também apresentava lipoma subcutâneo.

Epúlide acantomatosa - Ocorreu em um cão Cocker Spaniel e em duas cadelas Collie, com idades entre 5 e 14 anos, sempre com localização gengival. Segundo os registros, as epúlides, nas cadelas Collie, haviam sido excisadas cirurgicamente e recidivaram um ano depois. Uma das epúlides se infiltrou pelo osso alveolar e a outra coexistia com um adenocarcinoma de glândulas mucosas localizado no palato. No Cocker Spaniel, o tumor surgiu próximo ao quarto pré-molar superior e infiltrou-se até as vias aéreas superiores e órbita.

Ameloblastoma (adamantinoma) - Registraram-se 2 ameloblastomas, ambos gengivais, em uma gata com 12 anos de idade e em uma cadela SRD. No caso da gata, o tumor havia se infiltrado pelo osso alveolar.

Neoplasias malignas - Houve 35 casos de neoplasia oral maligna, o que representa $72,9 \%$ do total de neoplasias orais. Desses 35 casos, 21 (60\%) ocorreram em cães e $12(34,2 \%)$ em bovinos. A Tabela 4 apresenta a distribuição dos diferentes tipos histológicos de neoplasia oral maligna entre as espécies.

No caso dos cães, a incidência mais elevada ocorreu na faixa de 8 a 10 anos de idade e nos bovinos na faixa de 4 a 6 anos. A Tabela 5 traz a distribuição, segundo a idade, para os bovinos e os caninos. Em aves, foram diagnosticados um caso em galinha e um em papagaio, 
Tabela 4 - Distribuição segundo a espécie animal dos diferentes tipos histológicos de neoplasias orais malignas diagnosticadas pelo Serviço de Patologia do Hospital Veterinário da Universidade Federal do Paraná entre abril de 1974 e dezembro de 1995

\begin{tabular}{lcccccr}
\hline & \multicolumn{5}{c}{ Espécie animal } & Total \\
\cline { 2 - 5 } Tipo histológico & Cão & Bovino & Papagaio & Galinha & \\
\hline Carcinoma espinocelular & 5 & 10 & 1 & - & 16 \\
Hemangiossarcoma & 5 & 1 & - & - & 6 \\
Fibrossarcoma & 3 & & - & - & 3 \\
Melanocarcinoma & 2 & & - & - & 2 \\
Linfossarcoma & 1 & 1 & - & 1 & 3 \\
Mastocitoma & 1 & - & - & - & 1 \\
Carcinoma indiferenciado & 1 & - & - & - & 1 \\
Condrossarcoma & 1 & - & - & - & 1 \\
Adenocarcinoma de & & & & & \\
$\quad$ glândula mucosa & 1 & - & - & - & 1 \\
Carcinóide & 1 & - & - & - & 1 \\
\hline Total & 21 & 12 & 1 & 1 & 35 \\
\hline
\end{tabular}

Tabela 5 - Distribuição, segundo a idade do paciente, dos casos de neoplasias orais malignas em bovinos e caninos diagnosticadas pelo Serviço de Patologia do Hospital Veterinário da Universidade Federal do Paraná entre abril de 1974 e dezembro de 1995

\begin{tabular}{|c|c|c|c|}
\hline \multicolumn{2}{|c|}{ Bovinos } & \multicolumn{2}{|c|}{ Caninos } \\
\hline $\begin{array}{l}\text { Idade } \\
\text { (anos) }\end{array}$ & $\begin{array}{l}\text { Número } \\
\text { decasos }\end{array}$ & $\begin{array}{l}\text { Idade } \\
\text { (anos) }\end{array}$ & $\begin{array}{c}\text { Número } \\
\text { de casos* }\end{array}$ \\
\hline $\begin{array}{r}0-2 \\
2-4 \\
4-6 \\
6-8 \\
8-10 \\
10-12 \\
12-14 \\
14-16 \\
16-18 \\
18-20 \\
\text { Adulto** }\end{array}$ & $\begin{array}{l}2 \\
2 \\
2 \\
- \\
- \\
1 \\
1 \\
- \\
1 \\
- \\
3\end{array}$ & $\begin{array}{r}0-2 \\
2-4 \\
4-6 \\
6-8 \\
8-10 \\
10-12 \\
12-14\end{array}$ & $\begin{array}{l}1 \\
1 \\
3 \\
1 \\
6 \\
2 \\
1\end{array}$ \\
\hline Total & 12 & & 15 \\
\hline
\end{tabular}

*Seis casos de caninos não foram incluídos por seu registro não indicar a idade do paciente.

${ }^{* *}$ Casos de bovinos cujo registro só trazia a palavra adulto como indicação de idade.

ambos adultos. Entre os cães, os machos foram a maioria $(15$ casos - $71,4 \%)$. Nos bovinos, porém, as fêmeas é que predominaram ( 9 casos - 75\%). A Tabela 6 apresenta a freqüência das neoplasias malignas segundo o sexo dos pacientes.

$\mathrm{Na}$ Tabela 7 está discriminada a freqüência de neoplasias malignas segundo o sítio na cavidade oral. Os sítios mais atingidos foram as gengivas (para os cães) e a língua (para os bovinos). As Tabelas 8 e 9 trazem a distribuição das neoplasias por raça em cães e bovinos, respectivamente. Cães SRD e Pequineses foram os mais atingidos, bem como bovinos SRD e Holandeses.
Tabela 6 - Distribuição, segundo a espécie e o sexo dos pacientes, dos casos de neoplasias orais malignas diagnosticadas no Serviço de Patologia do Hospital Veterinário da Universidade Federal do Paraná entre abril de 1974 e dezembro de 1995

\begin{tabular}{lrrrrrr}
\hline & \multicolumn{3}{c}{ Espécie animal } & Total \\
\cline { 2 - 5 } Sexo & Cão & \multicolumn{1}{c}{ Bovino } & Papagaio Galinha & \\
\hline Machos & 15 & 3 & - & - & 18 \\
Fêmeas & 6 & 9 & - & 1 & 16 \\
Não especificado & - & - & 1 & - & 1 \\
\hline Total & 21 & 12 & 1 & 1 & 35 \\
\hline
\end{tabular}

Tabela 7 - Distribuição, segundo a espécie animal e o sítio na cavidade oral, dos casos de neoplasias orais malignas diagnosticadas no Serviço de Patologia do Hospital Veterinário da Universidade Federal do Paraná entre abril de 1974 e dezembro de 1995

\begin{tabular}{lcccccr} 
& \multicolumn{4}{c}{ Espécie animal } & Total \\
\cline { 2 - 5 } Sítio na cavidade oral & Cão & Bovino & Papagaio Galinha & \\
\hline Gengiva* & 9 & 1 & - & - & 10 \\
Lábio & 3 & - & - & - & 3 \\
Palato & 2 & - & - & - & 2 \\
Língua & 1 & 10 & 1 & 1 & 13 \\
Não especificado & 6 & 1 & - & - & 7 \\
\hline Total & 21 & 12 & 1 & 1 & 35 \\
\hline
\end{tabular}

*Inclui-se aqui um fibrossarcoma que ocupava simult: neamente a gengiva e o palato.

Tabela 8 - Distribuição, segundo a raça, dos casos de neoplasias orais malignas diagnosticadas em cães no Serviço de Patologia do Hospital Veterinário da Universidade Federal do Paraná entre abril de 1974 e dezembro de 1995

\begin{tabular}{lc}
\hline Raças & Número de casos \\
\hline SRD & 7 \\
Pequinês & 5 \\
Collie & 2 \\
Poodle & 2 \\
Dobermann & 2 \\
Schnauzer & 1 \\
Dálmata & 1 \\
Cocker Spaniel & 1 \\
\hline Total & 21 \\
\hline
\end{tabular}

SDR: sem raça definida

Tabela 9 - Distribuição, segundo a raça, dos casos de neoplasias orais malignas diagnosticadas em bovinos pelo Serviço de Patologia do Hospital Veterinário da Universidade Federal do Paraná entre abril de 1974 e dezembro de 1995

\begin{tabular}{lc}
\hline Raças & Número de casos \\
\hline SRD & 4 \\
Holandês & 4 \\
Charolês & 1 \\
Nelore & 1 \\
Gir & 1 \\
Guzerá & 1 \\
\hline Total & 12 \\
\hline
\end{tabular}

SDR: sem raça definida 
Carcinoma espinocelular - Tal neoplasia foi diagnosticada em 10 bovinos, em 5 cães e em 1 papagaio do gênero Amazona. As distribuições por raça e sexo para cães e bovinos estão, respectivamente, nas Tabelas $10 \mathrm{e}$ 11. Entre os cães, os machos predominaram, sendo os SDR os mais atingidos. Nos bovinos, as fêmeas foram a maioria, sendo mais atingidos os Holandeses e os SRD. Em bovinos, a incidência foi maior na faixa de 4 a 5 anos. Em cães, a incidência máxima ocorreu na faixa de 8 a 10 anos. Nesta espécie, 2 dos 5 casos de carcinoma espinocelular diagnosticados ocorreram nas gengivas e 1 no palato; os registros não especificavam a localização dos outros 2. Nos bovinos, 9 dos tumores surgiram na língua. Dois deles estendiam-se até a faringe e outros 2 ocupavam a faringe e o esôfago simultaneamente. Metástase em linfonodo foi registrada em um caso. Um dos registros mencionava que o animal em questão consumia samambaia (Pteridium aquilinum). O registro não apontava o sítio de ocorrência do décimo caso de carcinoma em bovino. O papagaio, que tinha 20 anos de idade, apresentava carcinoma espinocelular na língua.

Hemangiossarcoma - Ocorreram 5 casos de hemangiossarcoma em cães e 1 em bovino. A Tabela 12 traz a distribuição dos hemangiossarcomas em cães, segundo a raça e o sexo. A raça canina mais atingida foi a Pequinês. Houve 4 casos em machos e 1 em fêmea. A idade dos animais afetados variou de 6 a 13 anos. O único caso em bovino foi um hemangiossarcoma gengival numa bezerra Holandês, de três meses de idade.

Tabela 10 - Distribuição, segundo a raça e o sexo, dos casos de carcinoma espinocelular da cavidade oral diagnosticados em cães pelo Serviço de Patologia do Hospital Veterinário da Universidade Federal do Paraná entre abril de 1974 e dezembro de 1995

\begin{tabular}{lccc}
\hline \multirow{2}{*}{ Raça } & \multicolumn{2}{c}{ Sexo } & Total \\
\cline { 2 - 3 } & Machos & Fêmeas & \\
\hline SRD & 2 & 2 & 4 \\
Schnauzer & 1 & $\vdots$ & 1 \\
\hline Total & 3 & 2 & 5 \\
\hline
\end{tabular}

SDR: sem raça definida

Tabela 11 - Distribuição, segundo a raça e o sexo, dos casos de carcinoma espinocelular da cavidade oral diagnosticados em bovinos pelo Serviço de Patologia do Hospital Veterinário da Universidade Federal do Paraná entre abril de 1974 e dezembro de 1995

\begin{tabular}{lcccccccc}
\hline Sexo & & & \multicolumn{3}{c}{ Raça } & & & Total \\
\cline { 2 - 8 } & SRD & Holandês & Charolês & Nelore & Gir & Guzerá & \\
\hline Machos & 2 & - & - & - & 1 & - & 3 \\
Fêmeas & 2 & 2 & 1 & 1 & - & 1 & 7 \\
\hline Total & 4 & 2 & 1 & 1 & 1 & 1 & 10 \\
\hline
\end{tabular}

SDR: sem raça definida
Tabela 12 - Distribuição, segundo a raça e o sexo dos pacientes, dos casos de hemangiossarcoma da cavidade oral diagnosticados em cães pelo Serviço de Patologia do Hospital Veterinário da Universidade Federal do Paraná entre abril de 1974 e dezembro de 1995

\begin{tabular}{lcccc}
\hline Sexo & \multicolumn{3}{c}{ Raça } & Total \\
\cline { 2 - 4 } & Pequinês & SRD & Collie & \\
\hline Machos & 2 & 1 & 1 & 4 \\
Fêmeas & - & 1 & - & 1 \\
\hline Total & 2 & 2 & 1 & 5 \\
\hline
\end{tabular}

SDR: sem raça definida

Fibrossarcoma - Foi diagnosticado em 3 cães, sendo 1 Cocker Spaniel, 1 Pequinês (ambos machos) e 1 Dobermann (fêmea), com idades variando entre 10 e 13 anos. Um dos tumores localizava-se na gengiva e outro ocupava a gengiva e o palato. O registro não especificava o sítio do terceiro tumor.

Melanocarcinoma - Foi diagnosticado em 2 cães Poodle, 1 macho de 10 anos (com o tumor localizado na língua) e uma fêmea de 6 anos (com um tumor no lábio).

Linfossarcoma - Os registros mencionavam ocorrência de linfossarcoma em 3 animais. Em um cão SRD, macho, o tumor atingia das gengivas superiores até as vias aéreas. Em uma vaca Holandês e em uma galinha, atingia a língua. Em nenhum destes casos mencionavase o envolvimento de outros tecidos ou órgãos.

Mastocitoma - Havia referência a mastocitoma gengival num Pequinês, macho, de 6 anos.

Carcinoma indiferenciado - Foi diagnosticado no lábio de um Dálmata macho.

Condrossarcoma - Foi encontrado na cavidade oral de um Dobermann, macho, de 4 anos.

Adenocarcinoma de glândula mucosa - Ocorreu no palato da mesma cadela Collie citada na referência à epúlide acantomatosa.

Carcinóide - Foi diagnosticada em um cão Pequinês de 1 ano de idade. Este tumor foi enviado ao laboratório com a descrição de "neoplasia metastática no maxilar esquerdo". Não havia referência ao possível sítio primário da neoplasia.

\section{Discussão}

Incluíram-se a epúlide acantomatosa e o ameloblastoma (adamantinoma) na categoria especial de neoplasias benignas localmente agressivas. Esta categoria reflete o comportamento peculiar dessas neoplasias que originou debates entre os patologistas a respeito de sua efetiva classificação. O ameloblastoma, nos trabalhos de Dorn e Priester (1976) e White et al. (1985), figura como maligno. Misdorp (1987) já o classifica como tumor benigno localmente agressivo. Os outros autores consultados 
(Maretta et al., 1990; White, 1991; Harvey e Emily, 1993) classificam-no como benigno e, além disso, Maretta et al. (1990) afirmam que ele raramente faz metástases. A epúlide acantomatosa, por outro lado, é classificada como benigna por Misdorp (1987) e Harvey e Emily (1993). Estes últimos observam que a epúlide acantomatosa, embora não faça metástases, comporta-se localmente como um tumor maligno. Maretta et al. (1990) classificam-na de neoplasia benigna localmente agressiva e White (1991) propõe reclassificá-la como carcinoma basocelular, porém Harvey e Emily (1993) discordam. Por outro lado, White (1991), mesmo chamando a epúlide acantomatosa de carcinoma basocelular não a considera maligna. Quanto ao carcinoma basocelular cutâneo, Theilen e Madewell (1987a), em vista de seu curso clínico benigno, sugerem que passe a ser chamado de tumor das células basais. No presente trabalho, acompanhando o pensamento atual a respeito do carcinoma basocelular cutâneo e, por extensão, da epúlide acantomatosa, os autores também tendem a vê-los apenas como localmente agressivos.

Dorn e Priester (1976), usando dados coletados pelo Veterinary Medical Data Program (Califórnia) entre 1964 e 1974, pesquisaram 550 casos de neoplasia orofaríngea maligna (e localmente agressiva) ocorridos em cães, gatos, cavalos e bovinos. A maioria desses tumores ocorreu em cães ( 469 casos, ou $84 \%$ do total). No presente estudo, a maioria dos tumores malignos também ocorreu em cães ( 21 casos, ou $60 \%$ das neoplasias orais malignas). Em contrapartida, enquanto naquele trabalho os bovinos respondem por $31,2 \%$ dos casos de neoplasia oral maligna, Dorn e Priester (1976) encontraram apenas dois bovinos em sua pesquisa. Na literatura estrangeira, especialmente a norte-americana, os tumores orais em ruminantes não são considerados comuns (Andrews, 1985). No entanto, eles não são tão raros em países como o Brasil e o Quênia, provavelmente porque, nesses locais, os bovinos podem consumir o Pteridium aquilinum (Theilen e Madewell, 1987a, b).

Em animais selvagens e aves domésticas, as neoplasias orais também parecem não ser comuns. No Zoológico de Filadélfia, registraram-se apenas 33 casos entre 1908 e 1983. Destes, 31 casos ocorreram em mamíferos, 1 em réptil e 1 em ave. No Zoológico de San Diego, em 3.127 necropsias de mamíferos realizadas ao longo de 12 anos, encontrou-se apenas um adenocarcinoma oral, em Chrysocion brachyurus (Amand e Tinkelman, 1985). Na presente pesquisa, $6,2 \%$ de todos os casos de neoplasia oral ocorreram em mamíferos e aves selvagens e em aves domésticas.

Neste estudo, só se registrou um caso de neoplasia oral em gato, enquanto que, na pesquisa de Dorn e Priester (1976) os gatos perfaziam 9\% dos casos, provavelmente devido a maior popularidade desses mamíferos em outros países.
Os cães machos são mais propensos a neoplasias orais que as fêmeas (Dorn e Priester,1976; Theilen e Madewell, 1987a, b); a presente pesquisa confirma tal afirmação. No entanto, quanto aos bovinos, observou-se que as fêmeas foram mais atingidas pelas neoplasias orais. Tal constatação deve ser reflexo do sistema de criação que tende a preservar as fêmeas e descartar os machos.

As raças caninas de maior risco para neoplasias orais malignas são o Cocker Spaniel, o Poodle, o Pastor Alemão (Dorn e Priester, 1976; Todoroff e Brodey, 1979), o Weimaraner e o Boxer (Dorn e Priester, 1976; Theilen e Madewell, 1987a, b; Tholen e Hoyt, 1991). Na presente pesquisa, porém, a maior incidência foi observada nos cães SRD, seguidos pelos Pequineses. Quanto aos SRD, talvez seja reflexo do tamanho de sua população e do fato do HV-UFPR atender a muitos clientes de baixo poder aquisitivo e que usualmente não possuem cães de raças puras. No caso dos Pequineses, 2 casos de neoplasia oral maligna foram diagnosticados na década de 80 . Os outros 3 casos (além de 1 caso de neoplasia benigna) foram diagnosticados entre 1976 e 1978, época em que essa raça era muito popular na região de Curitiba. Estranhamente, os Boxers, neste trabalho, aparecem unicamente como portadores de neoplasia oral benigna, no caso a epúlide fibromatosa, com incidência alta (Maretta et al., 1990). Todas as neoplasias malignas encontradas em Boxers eram extra-orais. A alta freqüência de neoplasia oral maligna em bovinos SRD possivelmente se deve às mesmas razões que aquela dos cães SRD.

Neoplasias orais benignas e malignas são raras em cães com menos de 2 anos de idade (Patnaik et al., 1986). Na presente pesquisa apenas 2 dos 30 cães estudados tinham menos de 2 anos. Os tumores orais malignos, nos cães, ocorrem com maior freqüência dos 9 aos 11 anos (Bostock e Curtis, 1984). A incidência de neoplasia oral maligna nos cães aqui estudados é maior na faixa de 8 a 10 anos.

Entre as neoplasias orofaríngeas, o melanocarcinoma é o tumor maligno mais freqüente em cães, seguido pelo carcinoma espinocelular (não-tonsilar) e pelo fibrossarcoma (Bostock e Curtis, 1984). Na presente pesquisa, os tumores mais freqüentes nos cães foram o carcinoma espinocelular e o hemangiossarcoma, cada um com 5 casos, ou $23,8 \%$ do total de neoplasias malignas diagnosticadas em cães. Após vinham o fibrossarcoma com 3 casos $(14,2 \%)$ e o melanoma com apenas 2 casos (9,5\%). White et al. (1985), estudando 100 cães atendidos num período de 3 anos na Universidade de Cambridge, encontrraram dados semelhantes, sendo o carcinoma espinocelular a neoplasia oral mais freqüente, seguida pelo fibrossarcoma. O melanoma e o mastocitoma estavam em terceiro lugar em incidência.

Nos bovinos, segundo Andrews (1985), o carcinoma espinocelular é a neoplasia oral maligna mais freqüente. 
Nos cães, os sítios mais atingidos por tumores são as gengivas, a mucosa bucal, o palato e a língua (Tholen e Hoyt, 1990). Na presente pesquisa, as gengivas foram o local mais atingido pelos tumores no cão, seguidas pelos lábios (mucosa), palato e língua. Nos bovinos, o sítio de maior incidência foi a língua.

Neste estudo, encontraram-se 2 ameloblastomas, 1 dos quais em gato. Na Universidade da Pensilvânia, um estudo envolvendo 639 neoplasias orais benignas e malignas em gatos apontou o ameloblastoma respondendo por 12 casos ( $1,6 \%$ do total), sendo mais freqüente que a epúlide acantomatosa (Goldschmidt e Shofer, apud Harvey e Emily, 1993). O ameloblastoma costuma invadir e muitas vezes provocar reabsorção de osso alveolar (Theilen e Madewell, 1987a, b; Harvey e Emily, 1993).

O comportamento da epúlide acantomatosa, no presente estudo, corresponde bem àquele descrito na literatura. Essa neoplasia é muito invasiva e recidiva freqüentemente após excisão cirúrgica. É muito mais comum em cães do que em outras espécies (Madewell e Theilen, 1987a, b; White, 1991; Harvey e Emily, 1993).

O sítio de predileção dos carcinomas espinocelulares são as gengivas (White, 1991): Esses tumores tendem a ser muito invasivos (Tholen e Hoyt, 1990). O fibrossarcoma tem igual predileção pelas gengivas, especialmente as da arcada superior entre os dentes canino e quarto pré-molar (White, 1991) e pelo palato (Theilen e Madewell, 1987a, b). No presente estudo, a distribuição desses dois tumores na cavidade oral seguiu essas tendências; embora não fossem incluídas as neoplasias tonsilares, não se registrou nenhum caso de carcinoma espinocelular de tonsila.

Hemangiossarcoma, linfossarcoma e mastocitoma orais são considerados pouco comuns pela literatura. Considera-se que a cavidade oral não seja sítio primário para esses tumores (Maretta et al., 1990). Na Universidade da Pensilvânia, num estudo envolvendo 2.875 neoplasias orais benignas e malignas em cães, havia 16 casos de hemangiossarcoma $(0,2 \%$ do total) (Goldschmidt e Shofer, apud Harvey e Emily, 1993). Na presente pesquisa, o hemangiossarcoma não só foi um dos tumores mais freqüentes no cão, como jamais os registros traziam menção a um possível tumor primário em outro local.

O carcinóide é um tumor de células APUD (Amine Precursor Uptake and Decarboxylation). Foi descrito nos intestinos, pulmões e fígado de cães e no fígado de uma vaca (Patnaik et al., 1981). Em seres humanos, foi descrito na traquéia, mama, mediastino, trato gastrintestinal, ovário, esôfago e colo uterino (Patnaik et al., 1981). O estudo do carcinóide aqui mencionado ficou prejudicado pela ambigüidade do registro. É digno de nota, porém, que foi o único carcinóide dentre 960 neoplasias diagnosticadas no período estudado.

\section{Abstract}

Retrospective study of neoplasms of the oral cavity diagnosed between 1974 and 1995 at the Service of Animal Pathology, Veterinary Hospital, Federal University of Paraná, Curitiba, PR, Brazil

Among 960 neoplasms diagnosed in the Service of Animal Pathology of the Veterinary Teaching Hospital of the Federal University of Paraná, Brazil, 48 (5\%) were located in the oral cavity of 31 dogs, 13 bovine, 1 cat, 1 chicken, 1 lion and 1 parrot. Thirty five tumors (72.9\%) were malignant (5 squamous cell carcinomas, 5 hemangiosarcomas, 3 fibrosarcomas, 2 melanocarcinomas, 1 lymphosarcoma, 1 mastocitoma, 1 mucous gland adenocarcinoma, 1 undifferentiated carcinoma and 1 carcinoid. Thirteen tumors $(27.1 \%)$ were benign, with the addition of 5 cases considered as local aggressive rather than malignant ( 3 acanthomatous epulides and 2 ameloblastomas). The remaining benign tumors were 5 cases of fibromatous epulides and 3 cases of papilomas.

Key words: oral cavity tumors; neoplasia; veterinary pathology

\section{Referências bibliográficas}

Amand WB, Tinkelman CL 1985. Oral disease in captive wild animals, p. 289-311. In CE Harvey, Veterinary dentistry, W.B. Saunders, Philadelpha.

Andrews AH 1985. Acquired diseases of the teeth and mouth in ruminants, p. 256-271. In CE Harvey, Veterinary dentistry, W.B. Saunders, Philadelphia.

Bostock DE, Curtis R 1984. Comparison of canine oropharyngeal malignancy in various geographical locations. Vet Record 114: 341-342.

Bittencourt RH et al. 1994. Ocorrência de neoplasias em cães e gatos no HOVET-FCAP. Anais do XXIII Congresso Brasileiro de Medicina Veterinária, Pernambuco, p. 51.

Dorn CR, Prister WA 1976. Epidemiologic analysis of oral and pharyngeal cancer in dogs, cats, horses and cattle. J Am Vet Med Assoc 169: 1202-1206.

Harvey CE, Emily PP 1993. Small Animal Dentistry. St. Louis, Mosby, $626 \mathrm{pp}$.

Maretta SM et al. 1990. Surgical management of oral neoplasia, p. 96-120. In MJ Bojrab e M Tholen (eds.), Small Animal Oral Medicine and Surgery, Lea \& Febiger, Philadelphia.

Misdorp W 1987. The impact of pathology on the study and treatment of cancer, p.53-70. In GH Theilen e BR Madelwell (eds.), Veterinary Cancer Medicine, 2ed., Lea \& Febiger, Philadelphia.

Patnaik AK et al. 1981. Canine hepatic carcinoids. Vet Pathol 18: $445-453$.

PatnaikAK et al. 1986. A clinico-pathological and ultrastructural siudy of undifferentiated malignant tumors of the oral cavity in dogs. Vet Pathol 23: 170-175.

Theilen GH, Madewell BR 1987. Tumors of the digestive tract, p. 499-534. In GH Theilenm e BR Madewell (eds.), Veterinary Cancer Medicine, 2nd ed., Lea \& Febiger, 
Philadelphia.

Theilen GH, Madewell BR 1987 . Tumors and tumor-like conditions of epithelial origin, p. 40-266. In GH Theilen e BR Madewell (eds.), Veterinary Cancer Medicine, 2nd ed., Lea \& Febiger, Philadelphia.

Tholen M, Hoyt Jr RF 1990. Oral pathology, p. 240-266. In MJ Bojrab e M Tholen (eds.), Small Animal Oral Medicine and Surgery, Lea e Febiger, Philadelphia.
Todoroff RJ, Brodey RS 1979. Oral and pharyngeal neoplasia in the dog: a retrospective survey of 361 cases. J Am Vet Med Assoc 175: 567-571.

White RAS et al. 1985. Clinical staging for oropharyngeal malignancies in the dog. J Small Anim Practice 26: 581594.

White RAS 1991. The alimentary system, p. 237-263. In RAS White, Manual of Small Animal Oncology, BSAVA. 